\title{
Wiktor Stent Implantation in Patients with Restenosis Following Balloon Angioplasty of a Native Coronary Artery
}

\author{
Peter P. de Jaegere, MD, Patrick W. Serruys, MD, Michel Bertrand, MD, \\ Volker Wiegand, MD, Gisbert Kober, MD, Jean Francois Marquis, MD, \\ Bernard Valeix, MD, Rainer Uebis, MD, and Jan Piessens, MD
}

\begin{abstract}
Intracoronary stenting has been introduced as an adjunct to balloon angioplasty aimed at overcoming its limitations, namely acute vessel closure and late restenosis. This study reports the first experience with the Wiktor ${ }^{\mathrm{TM}}$ stent implanted in the first 50 consecutive patients. All patients had restenosis of a native coronary artery lesion after prior balloon angioplasty. The target coronary artery was the left anterior descending artery in 26 patients, the circumflex artery in 7 patients and the right coronary artery in 17 patients. The implantation success rate was $98 \%$ (49 of 50 patients). There were no procedural deaths. Acute or subacute thrombotic stent occlusion occurred in 5 patients (10\%). All 5 patients sustained a nonfatal acute myocardial infarction. Four of these patients underwent recanalization by means of balloon angioplasty; the remaining patient was referred for bypass surgery. A major bleeding complication occurred in 11 patients (22\%): groin bleeding neces-
\end{abstract} sitating blood transfusion in 6 , gastrointestinal bleeding in 3 and hematuria in 2 . Repeat angiography was performed at a mean of $5.6 \pm 1.1$ months in all but 1 patient undergoing implantation. Restenosis, defined by a reduction of $\geq 0.72$ $\mathrm{mm}$ in the minimal luminal diameter or a change in diameter stenosis from $<$ to $\geq \mathbf{5 0} \%$, occurred in 20 (45\%) and 13 (29\%) patients, respectively. In this first experience, the easiness and high technical success rate of Wiktor stent implantation are overshadowed by a high incidence of subacute stent occlusion and bleeding complications. Although direct comparison with balloon angloplasty regarding the incidence of subsequent restenosis

From the Catheterization Laboratory and Laboratory for Quantitative Angiographic Analysis (Cardialysis), Thoraxcenter, Rotterdam, the Netherlands; Department of Cardiology, Hôpital Cardiologique, Lille, France; Department of Cardiology, Georg August Universität, Göttingen, Germany; Department of Cardiology, Klinikum der J.W. Goethe Universität, Frankfurt, Germany; Department of Cardiology, University of Ottawa Heart Institute, Ottawa, Canada; Department of Cardiology, Clinique la Casamance, Marseille, France; Medical Clinic I RWTH, Aachen, Germany; and the Department of Cardiology, University Hospital Gasthuisberg, Leuven, Belgium. Manuscript received August 5, 1991; revised manuscript received and accepted November 1, 1991.

Address for reprints: Patrick W. Serruys, MD, Catheterization Laboratory and Laboratory for Quantitative Angiographic Analysis (Cardialysis), Thoraxcenter, Erasmus University, P.O. Box 1738, 3000 DR Rotterdam, the Netherlands. rate is not possible, the experience reported in this study compares favorably with data reported in other published reports.

(Am J Cardiol 1992;69:598-602)

$P$ ercutaneous transluminal coronary balloon angioplasty (PTCA) has established its role as a nonsurgical revascularization procedure in selected patients with obstructive coronary artery disease. Gained operator experience and improved catheter technology has resulted in a high immediate success rate and a low incidence of complications. ${ }^{1}$ These favorable initial results are compromised by the unpredictable problem of late restenosis, occurring in 20 to $40 \%$ of the patients after successful PTCA. ${ }^{2-5}$ Furthermore, there is some evidence that the recurrence of subsequent restenosis after repeat PTCA increases with the number of repeat interventions. ${ }^{6-9}$ Intracoronary stents are one of the new technologies that (along with pharmacologic interventions, atherectomy and laser techniques) have entered clinical testing to address this problem. ${ }^{10} \mathrm{Re}$ cently, the first experience with the self-expandable Wallstent ${ }^{\circledR}$ and the balloon expandable Palmaz-Schat ${ }^{\circledR}$ stent have been reported. ${ }^{11,12}$ The stent used in this study is the Medtronic Wiktor ${ }^{\mathrm{TM}}$ stent which, in contrast to the Wallstent and the Palmaz-Schatz stent, is not a mesh-made stainless steel device but a coil-like prosthesis made of a single loose interdigitating tantalum wire formed into a sinusoidal wave and configured as a helix. This loose configuration may offer less scaffolding properties compared with the other 2 stents. Therefore, the purpose of this study was to assess the immediate and long-term clinical and angiographic results following Wiktor stent implantation in the first 50 consecutive patients with restenosis following balloon angioplasty of a native coronary artery lesion.

\section{METHODS}

Patients: The study group consisted of the first 50 consecutive patients in whom a single Wiktor stent implantation was attempted (Table I). All patients had recurrence of angina with objective evidence of ischemia due to restenosis of a native coronary artery lesion following PTCA. A first restenosis was documented in 33 patients, a second restenosis in 13 patients and a third in 4 patients. The dilated and stented coronary artery was the left anterior descending artery in 26 patients (52\%), 
the circumflex artery in 7 patients $(14 \%)$ and the right coronary artery in 17 patients (34\%). A written informed consent was required for every patient. The study protocol was approved by the ethics committee of the individual hospitals. The stents used in this study are shown in Figures 1 and 2. They are described in detail elsewhere as well as the drug protocol associated with stent implantation. ${ }^{11,13}$ The angiograms were analyzed using automated edge-detection as previously described. ${ }^{14,15}$

End points: Primary clinical end points were death, myocardial infarction, bypass surgery or repeat intervention and bleeding complications. All deaths were considered cardiac, unless an unequivocal noncardiac cause could be established. The primary angiographic end point was the change in minimal luminal diameter at the dilated and stented segment at 6 months relative to the baseline. If a revascularization procedure involving the stented segment had been performed before 6 months repeat angiography, the last angiogram before this intervention was used to obtain follow-up values, irrespective of the timing of repeat PTCA (hours, days or weeks). The secondary angiographic end point was the incidence of restenosis defined according to the following criteria: First, a continuous approach was used in which restenosis was defined by a reduction of $\geq 0.72$ $\mathrm{mm}$ in the minimal luminal diameter at follow-up. This change in minimal luminal diameter has been found to be a reliable indicator of angiographic progression of vessel narrowing. This value takes into account the limitations of coronary angiographic measurements and represents twice the long-term variability of repeat measurements of a coronary artery obstruction with the Cardiovascular Angiographic Analysis System. ${ }^{15}$ Second, a categorical approach was used in which an increase in diameter stenosis of $<50 \%$ immediately after stent implantation to $\geq 50 \%$ at follow-up was used as a cutoff point to define restenosis. This criterion was selected since common clinical practice has continued to express lesion severity as a percentage of stenosis.

Statistics: Values obtained by quantitative angiographic analysis are expressed as mean \pm SD. The means for each angiographic variable before PTCA, after PTCA, immediately after stent implantation and at follow-up were compared by analysis of variance. If significant differences were found, 2-tailed $t$ tests were applied to paired data. A statistical probability of $<0.05$ was considered significant. In the patient with the miss-

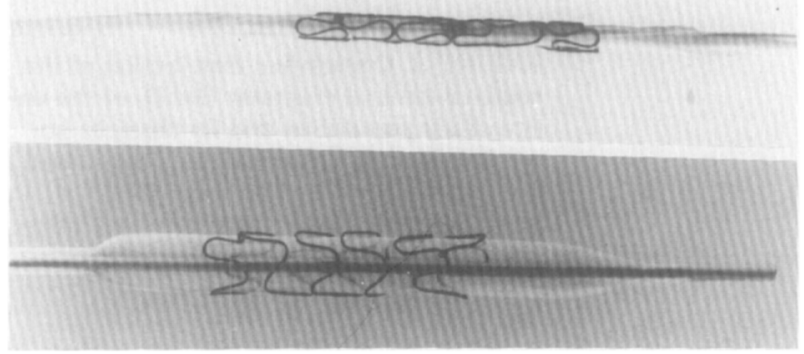

FIGURE 1. Medtronic Wiktor stent mounted on a conventional polyethylene balloon when deflated (top panel) and inflated (bottom panel).

TABLE I Clinical Characteristics

No. of patients

Age (mean $\pm S D, y r s)$

Men

Functional class*

I

II

III

IV

Systemic hypertension

Cholesterol > $200 \mathrm{mg} \%$

Cigarette smokers

Diabetes mellitus ${ }^{\dagger}$

50

$55 \pm 9$

44

10

6

14

20

7

7

17

6

ardiovascular Society.

†All non-insulin-dependent.

ing follow-up angiogram and no clinical evidence of restenosis, imputation of the minimal luminal diameter at follow-up was performed: the minimal luminal diameter at follow-up of this patient was calculated by subtracting the mean change of the minimal luminal diameter of the entire study population from the value of the minimal luminal diameter immediately after stent implantation of this particular patient.

\section{RESULTS}

Clinical results: The implantation success rate was 98\% (49 of 50 attempted implants). In 1 patient, the stent could not be delivered at the target site due to the inability to cross a tortuous proximal right coronary artery. There were no procedural related deaths. However, subacute thrombotic stent occlusion occurred in 5 patients $(10 \%)$ while still in the hospital. The occurrence to this thrombotic event with respect to the implantation was as follows: day $0=1$ patient, day $1=1$

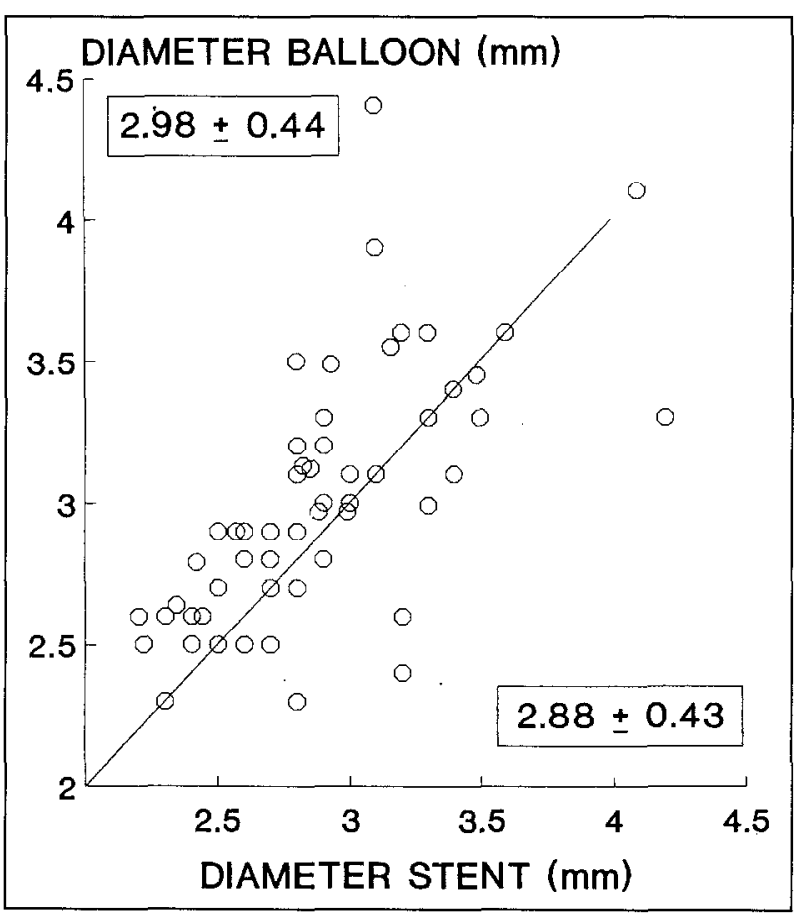

FIGURE 2. Difference between the mean diameter of the balloon when fully inflated and stented segment immediately after implantation. 


\begin{tabular}{|c|c|c|c|c|c|}
\hline & \multirow[b]{2}{*}{$\begin{array}{l}\text { Before } \\
\text { PTCA }\end{array}$} & \multirow[b]{2}{*}{$\begin{array}{l}\text { After } \\
\text { PTCA }\end{array}$} & \multirow[b]{2}{*}{$\begin{array}{l}\text { After Stent } \\
\text { Implantation }\end{array}$} & \multicolumn{2}{|c|}{ At Follow-Up } \\
\hline & & & & $\begin{array}{l}\text { All Pts. } \\
(n=49)\end{array}$ & $\begin{array}{l}\text { Pts. Without } \\
\text { Subacute } \\
\text { Occlusion } \\
(n=44)\end{array}$ \\
\hline $\begin{array}{l}\text { Minimal luminal diameter } \\
(\mathrm{mm})\end{array}$ & $1.09 \pm 0.26$ & $1.80 \pm 0.32$ & $2.45 \pm 0.35$ & $1.59 \pm 0.79$ & $1.78 \pm 0.60$ \\
\hline Diameter stenosis $(\%)$ & $\stackrel{61 \pm 9}{-p<}$ & $1 \stackrel{34 \pm 11}{-}$ & $18 \pm 7$ & $\begin{array}{r}45 \pm 25 \\
0001 \\
0001\end{array}$ & $39 \pm 18$ \\
\hline
\end{tabular}

patient, day $4=1$ patient, and day $5=2$ patients. Four patients underwent recanalization by means of conventional balloon angioplasty. In 1 of these patients adjunctive thrombolytic therapy was used. In the remaining patient, repeat PTCA was not attempted because retrospective analysis of the implantation angiogram revealed incomplete stent expansion. This patient was referred for emergency bypass surgery. All 5 patients sustained a nonfatal acute myocardial infarction (mean \pm $\mathrm{SD}$, creatine phosphokinase $1,797 \pm 1,849 \mathrm{U} /$ liter). A major bleeding complication occurred in 11 patients (22\%): 6 patients sustained a groin hematoma necessitating blood transfusion, 3 patients had a gastrointestinal bleeding ( 2 duodenal ulcers, 1 intestinal polyps) and 2 patients had hematuria. One patient had an infected pharyngeal hematoma caused by acenocoumarol therapy at 3 months after implant.

During follow-up, 1 patient died 3 months after stent implantation following prostate surgery. This patient had a history of bypass surgery, mitral valve replacement and multiple PTCA's. Because of severe disabling heart failure, he was screened for heart transplantation. During this period, a prostate adenoma was found and surgically removed. The second day after surgery, he died because of heart failure. Although necropsy was not performed, death was not considered stent-related because there was no clinical evidence of restenosis. No patient sustained an acute myocardial infarction. Three patients $(6 \%)$ underwent bypass surgery (2 patients 3.5 months and 1 patient 6 months after the stent implantation). There was a significant improvement in functional class at 6-month follow-up. Before stent implantation, 10 patients were in class I, 6 in class II, 14 in class III and 20 in class IV, according to the Canadian Cardiovascular Society. At follow-up, 34 patients were in class I, 9 in class II, 4 in class III and 3 in class IV.

Angiographic results: Wiktor stent implantation resulted in a further significant increase in minimal luminal diameter, from $1.80 \pm 0.32 \mathrm{~mm}$ immediately following balloon angioplasty to $2.45 \pm 0.35 \mathrm{~mm}$ immediately after stent implantation. This was associated with a reduction in diameter stenosis from $34 \pm 11$ to $18 \pm$ $7 \%$ (Table II). There was no change in the reference diameter before and after PTCA (2.81 \pm 0.48 and 2.80 $\pm 0.48 \mathrm{~mm}$, respectively, $p=$ not significant). However, there was a significant increase after stent implantation $(2.98 \pm 0.42 \mathrm{~mm}, \mathrm{p}<0.00001)$, which was confirmed at follow-up $(2.91 \pm 0.55 \mathrm{~mm})$. In most patients the measured diameter of the balloon when fully inflated was higher than the measured diameter of the stent (Figure 2). During maximal inflation the mean diameter of the balloon for the total study group was $2.98 \pm 0.44 \mathrm{~mm}$. The mean diameter of the stented segment immediately after implantation was $2.88 \pm 0.43 \mathrm{~mm}$. This implies a recoil of $0.10 \pm 0.36 \mathrm{~mm}(\mathrm{p}<0.03)$.

Repeat angiography was performed in all patients with successful stent implantation (49 patients), except in 1, who died 3 months after stent implantation follow-

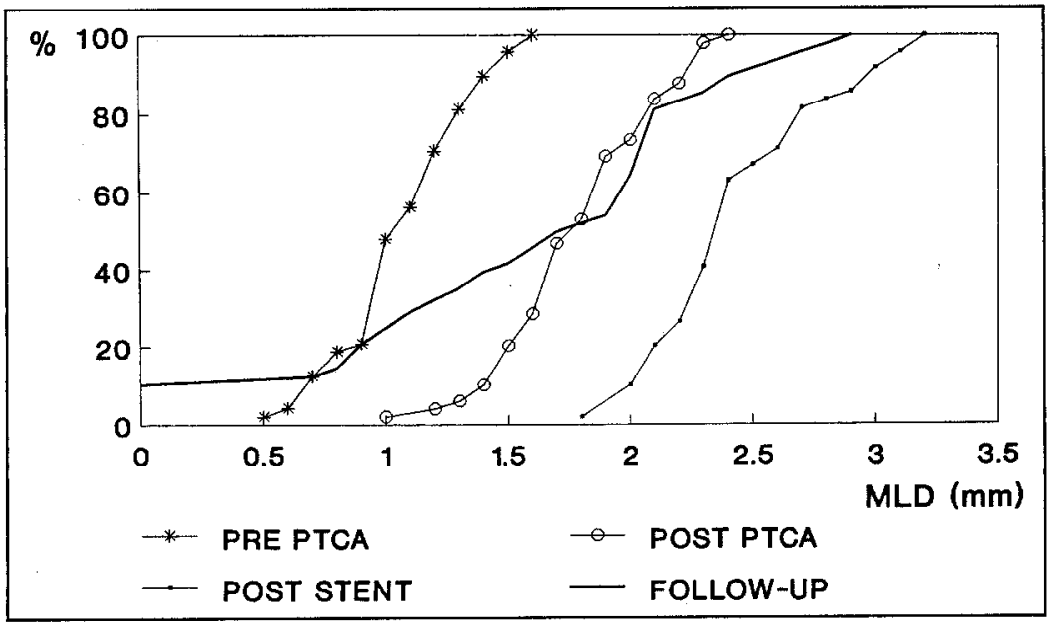

FIGURE 3. Cumulative distribution of the minimal huminal diameter (MLD) of the entire study population and its changes immediately after balloon angioplasty, stent implantation and at follow-up. PTCA = percutaneous transtuminal coronary angioplasty. 
ing prostate surgery. There was no clinical evidence of restenosis in this patient. The mean time interval between stent implantation and control study was $5.6 \pm$ 1.1 months. Overall, the minimal luminal diameter was found to have decreased from $2.45 \pm 0.35$ to $1.59 \pm$ $0.79 \mathrm{~mm}$ ( $\mathrm{p}<0.00001$ ). The percentage of stenosis had increased from $18 \pm 7$ to $45 \pm 25 \%$ ( $p<0.0001$, Table II). When only patients without clinical evidence of subacute vessel closure during hospital stay (44 patients) were included, the minimal luminal diametcr and percentage of stenosis were $1.78 \pm 0.60 \mathrm{~mm}$ and $39 \pm$ $18 \%$, respectively (Table II). Figure 3 displays the cumulative distribution of the minimal luminal diameter and its changes (immediately after balloon angioplasty and stent implantation and at follow-up). The additional increase in minimal luminal diameter immediately after stent implantation is lost at follow-up.

The incidence of restenosis depended on the definition used. When a change of $\geq 0.72 \mathrm{~mm}$ in minimal luminal diameter was used as the criterion, restenosis was observed within the stent in 19 patients $(43 \%)$ and in the segment immediately distal to the stent in $1(2 \%)$ of the $\mathbf{4 4}$ patients without clinical evidence of subacute stent occlusion during hospital stay. Of these 44 patients, the percentage of stenosis at follow-up had increased to $\geq 50 \%$ within the stent in $12(27 \%)$ and in the segment distal to the stent in $1(2 \%)$. Therefore, the total restenosis rate was $45 \%$ according to the $0.72 \mathrm{~mm}$ criterion and $29 \%$ according to the $50 \%$ diameter stenosis criterion (Figure 4).

\section{DISCUSSION}

Clinical results: Intracoronary stents are currently being tested in clinical practice to reduce the incidence of acute vessel occlusion and restenosis following balloon angioplasty. Several stents are available, each with its own specific design, composition and physicochemical behavior once implanted. Implanting foreign body material implies an increased risk for acute thrombosis. Therefore, a stringent anticoagulation protocol is mandatory. Despite the protocol previously described, a thrombotic subacute stent occlusion occurred in 5 patients $(10 \%)$. Three of these patients did not receive dextran and 1 patient was not properly treated with acenocoumarol. Of the 44 patients without clinical evidence of subacute stent occlusion during hospital stay, only $6(14 \%)$ did not receive dextran. Unfortunately, since central assessment of anticoagulation was not performed, the exact role of the failure of anticoagulation with respect to subacute thrombotic stent occlusion cannot be elucidated. Although it is tempting to speculate that suboptimal anticoagulation may be the main cause of stent occlusion, other pathophysiologic mechanisms also may be involved. Before stent implantation, balloon angioplasty is performed to facilitate stent delivery. The disruptive action of the balloon may cause intimal dissection, which in turn may be the primary cause of an ensuing thrombotic event. Furthermore, patients' related factors such as acute ischemic syndromes, procedurerelated factors such as technical difficult stent implantation and angiographic related factors such as small vessel size and total occlusion may predispose to throm- botic stent occlusion. In this study group, no difference in vessel size was seen in patients with and without subacute stent occlusion (reference diameter $2.8 \pm 0.4$ and $2.8 \pm 0.5 \mathrm{~mm}$, respectively ( $\mathrm{p}=$ not significant $)$. The reported subacute stent occlusion rate compares very favorably with the initial Wallstent experience $(20 \%$ in 105 patients), but is similar to the subacute occlusion rate following the extended Wallstent experience (12\% in an additional 160 patients). ${ }^{11,16}$ However, it contrasts sharply with the incidence of $0.6 \%$ following PalmazSchatz stent implantation. ${ }^{12}$ True comparison is not possible because the studies differ in methods for patient selection, indication for stent implantation and type of vessel stented. Furthermore, the very low incidence of $0.6 \%$ reported by Schatz, has not been confirmed by a recent study, using the same device. ${ }^{17}$ In this latter study, an incidence of $16 \%$ is reported. Probably, the incidence of subacute stent occlusion may be reduced by a more detailed coagulation monitoring. Indeed, recent work indicates that measurement of prothrombin fragment $1+2$ and its changes after stent implantation may be predictive for subacute thrombotic stent occlusion..$^{18}$

Another matter of concern is the risk of bleeding, inherently associated with aggressive anticoagulation. The incidence of bleeding is negligible after PTCA but is substantial when a combination of intravenous and

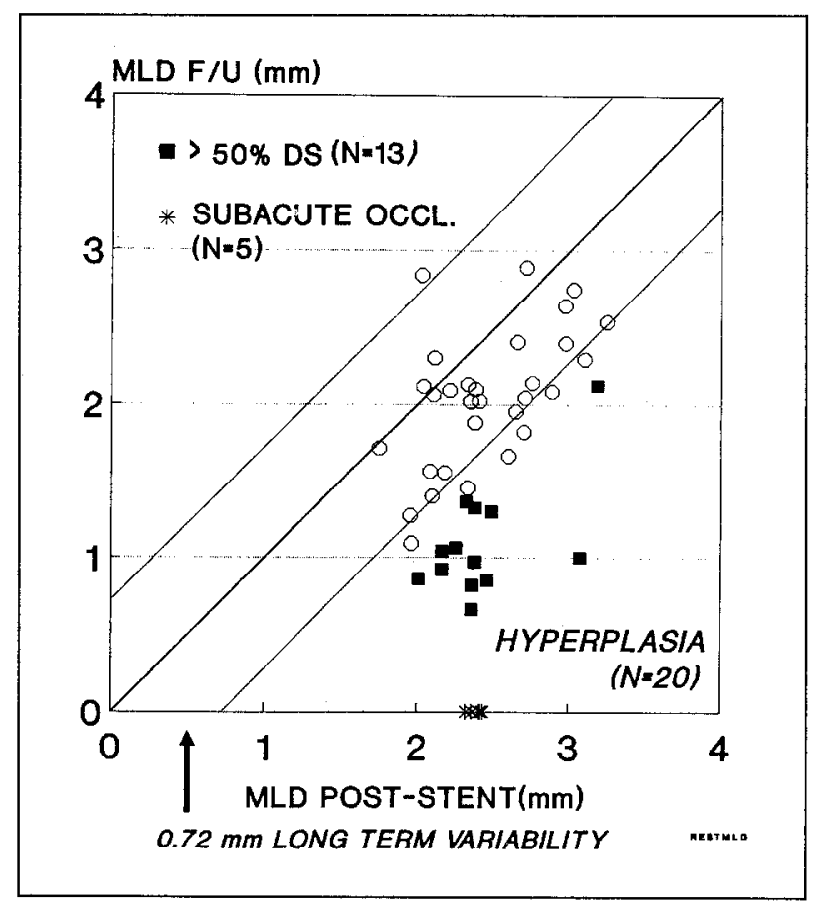

FIGURE 4. Changes in the minimal luminal diameter (MLD) at follow-up $(F / U)$ after Wiktor stent implantation in native coronary arteries. The diameter of each segment immediately after stent implantation is plotted against the diameter at follow-up. The lines on each side of the line of identity (diagonal) represent twice the variability ( $95 \%$ confidence interval) of duplicate measurements (a change of $\geq 0.72 \mathrm{~mm}$ ). Of the 49 patients, $5(10 \%)$ sustained a subacute stent occlusion (OCCL.) $(X$-axis). In the remaining 44 patients, restenosis was observed in 20 patients $(45 \%$ ) according to the $0.72 \mathrm{~mm}$ criterion and in 13 patients $(29 \%)$ according to the $50 \%$ diameter stenosis (DS) criterion. 
oral anticoagulant drugs are administered. ${ }^{12}$ The most serious bleeding complication is intracranial hemorrhage, which occurred in 1 of 174 patients following Palmaz-Schatz stent implantation and in 3 of 265 patients following Wallstent implantation. ${ }^{12,16}$ Another aspect to be considered regarding anticoagulation is the longer period of hospitalization, which in turn increases the overall costs of the procedure. ${ }^{19}$ In this study, the hospital stay was (mean $\pm \mathrm{SD}$ ) $11.8 \pm 7.4$ days for the entire study population.

Angiographic results: The smaller mean diameter of the stented segment $(2.88 \pm 0.43 \mathrm{~mm})$ compared with the measured diameter of the fully expanded balloon $(2.98 \pm 0.44 \mathrm{~mm})$ suggests some recoil of the stented segment. This minimal recoil appears to be a true phenomenon since the accuracy and the precision of the quantitative coronary angiography system used in this study is -30 and $90 \mu$, respectively. ${ }^{15}$ Furthermore, recoil has also been observed, although to a larger extent, after Wiktor stent implantation in Yorkshire pigs. ${ }^{20}$ The more pronounced recoil $(10 \%)$ observed in the animal model compared with recoil observed in this study (3\%) may be explained by the fact that in the former animal study the stent was implanted in normal coronary arteries. All these angiographic data indicate that in contrast to balloon angioplasty, where recoil amounting to $50 \%$ has been documented, the Wiktor stent appropriately scaffolds the vessel. ${ }^{21}$

Restenosis and recurrence of restenosis remains the major limitation of (repeat) PTCA. Whether intracoronary stents can address this issue appropriately is still unknown. There are some encouraging data from preliminary reports..$^{22,23}$ However, firm conclusions cannot be drawn, since these data stem from nonrandomized studies in which single and multiple stent implantations have been performed in both native coronary arteries and venous bypass grafts have been performed in patients with either acute ischemic syndromes or stable angina for a variety of indications (primary stent implantation, restenosis, bail out). Patients undergoing repeat PTCA appear to be at a higher risk for recurrence of restenosis after repeat intervention. The angiographic documented restenosis rate increases from $34 \%$ after a second dilatation to $40 \%$ after a fourth dilatation. 6,9 This is probably an underestimation of the actual incidence owing to incomplete angiographic follow-up. Repeat angiography is mandatory in all patients, even in patients free of angina at follow-up since approximately $25 \%$ of the patients with restenosis are asymptomatic. ${ }^{24}$ Taking into account the limitations of these nonrandomized observational studies, as is done in this study, the incidence of $29 \%$ restenosis using the $50 \%$ diameter stenosis criterion compares favorably with data from those former studies. Indeed, randomized studies are needed to define the exact role of intracoronary stenting in the prevention of (recurrent) restenosis.

Acknowledgment: We greatly acknowledge MarieAngèle Morel for performing the quantitative analysis and for assisting in the database management and statistical analysis.

\section{REFERENCES}

1. Detre K, Holubkov R, Kesley S, Cowley M, Kent K, Williams D, Myler R, Faxon D, Holmes D Jr, Bourassa M, Block P. Gosselin A. Bentivoglio L, Leatherman L, Dorros G, King SB III, Galichia J, Al-Bassam M, Leon M, Robertson T, Passamani E, and co-investigators of the National Heart, Lung, and Blood Institute's Percutaneous Transluminal Coronary Angioplasty Registry. Percutaneous transluminal coronary angioplasty in 1985-1986 and 1977-1981. N Engl J Med 1988;318:265-270.

2. Serruys PW, Luijten HE, Beatt KJ, Geuskens R, de Feyter PJ, van den Brand $M$, Rciber JHC, ten Katen HJ, van Es GA, Hugenholtz PG. Incidence of resteno" sis after successful coronary angioplasty: a time-related phenomenon. Circulation 1988;77:361-371.

3. Leimgruber PP, Roubin GS, Hollman J, Cotsonis GA, Meier B, Douglas JS, King SB III, Gruentzig AR. Restenosis after successful coronary angioplasty in patients with single-vessel disease. Circulation 1986;73:710-717.

4. Levine S, Ewel CJ, Rosing DR, Kent KM. Coronary angioplasty: clinical and angiographic follow-up. $A m J$ Cardiol 1985;55:673-676.

5. Nobuyoshi M, Kimura T, Nosaka H, Mioka S, Ueno K, Yokoi H, Hamasaki $\mathrm{N}$, Horiuchi $\mathrm{H}$, Ohishi $\mathrm{H}$. Restenosis after successful percutaneous transluminal coronary angioplasty: serial angiographic follow-up of 229 patients. $J$ Am Coll Cardiol 1988;12:616-623.

6. Williams D, Gruentzig A., Kent K, Detre K, Kelsey S, To T. Efficacy of repeat percutaneous transluminal coronary angioplasty for restenosis. Am I Cardiol 1984;53:32C-35C.

7. Meier B, King SB III, Gruentzig A, Douglas J, Hollman J, Ischinger T, Galan K, Tankersley R. Repeat coronary angioplasty. I Am Coll Cardiol 1984; 4:463-466.

8. Black A, Anderson V, Roubin G, Powelson S, Douglas J, King S III. Repeat coronary angioplasty: correlates of a second restenosis. J Am Coll Cardiol 1988;11:714-718.

9. Teirstein P, Hoover C, Ligon R, Giorgi L, Rutherford B, McConahay D, Johnsom W, Hartzler G. Repeat coronary angioplasty: efficacy of a third angioplasty for a second restenosis. I Am Coll Cardiol 1989;13:291-296.

10. Sigwart U, Puel J, Mirkovitch V, Joffre F, Kappenberger L. Intravascular stents to prevent occlusion and restenosis after transluminal angioplasty. $N$ Engl $J$ Med 1987;316:701-706.

11. Serruys PW, Strauss B, Beatt K, Bertrand M, Puel J, Rickards A, Meier B, Kappenberger L, Goy J, Sigwart U. Angiographic follow-up after placement of a self-expanding coronary-artery stent. N Engl J Med 1991;324:13-17.

12. Schatz R, Baim D, Leon M, Ellis S, Goldberg S, Hirsfeld J, Cleman.M, Cabin H, Walker C, Stagg J, Buchbinder M, Teirstein P, Topol E, Savage M, Perez J, Curry R, Whitworth H, Sousa E, Tio F, Almagor Y, Ponder R, Penn I, I eonard B, I evine S, Fish D, Palmaz J. Clinical experience with the PalmazSchatz coronary stent. Initial results of a multicenter study. Circulation 1991; 83:148-161.

13. Serruys PW, de Jaegere $P$, Bertrand $M$, Kober G, Marquis JF, Piessens, Uebis R, Valeix B, Wiegand V. Morphologic change in coronary artery stenosis with the Medtronic Wiktor stent: initial results from the core laboratory for quantitative angiography. Cath Cardiovasc Diagn 1991;24:237-245.

14. Reiber J, Serruys PW, Sluger C. Cardiovascular Angiography System (CASS). In: Quantitative Coronary and Left Ventricular Cineangiography: Methodology and Clinical Applications. Boston: Nijhoff, 1986:62-87.

15. Reiber JHC, Serruys PW, Kooijman CJ. Assessment of short-, medium-, and long-term variations in arterial dimensions from computer-assisted quantitation of coronary cineangiograms. Circulation 1985;71:280-288.

16. Strauss B, Serruys PW, Bertrand M, Puel J, Meier B, Goy JJ, Kappenberger L, Rickards A, Sigwart U. Quantitative angiographic follow-up of the coronary Wallstent in native vessels and bypass grafts. European Experience March 1986-March 1990. Am J Cardiol 1992;69:475-481.

17. Haude M, Erbel R, Straub U, Dietz U, Meyer J. Short and long-term results after intracoronary stenting in human coronary arteries: monocenter experience with the balloon-expandable Palmaz-Schatz stent. Br Heart $J$ 1991;66:337-346. 18. Hafner G, Swars H, Erbel R, Ehrenthal W, Rupprecht H, Lotz J, Meyer J, Prellwitz W. Monitoring prothrombin fragment $1+2$ and coagulation factor II to avoid subacute occlusion after coronary stenting; in press.

19. Dick R, Popma J, Muller D, Burek K, Topol E. In-hospital costs associated with new percutaneous coronary devices. Am J Cardiol 1991;68:879-885A.

20. Van der Giessen WJ, Serruys PW, van Beusekom HMM, van Woerkens LJ, van Loon H, Loe Kie Soei, Strauss BH, Verdouw P. Coronary stenting with a new, radiopaque, balloon-expandable endoprosthesis in pigs. Circulation 1991;83: $1788-1798$.

21. Rensing BJ, Hermans RM, Beatt KJ, Laarman GJ, Suryapranata $H$, van den Brand M, de Feyter PJ, Serruys PW. Quantitative angiographic assessment of elastic recoil after percutaneous transluminal coronary angioplasty. Am $J$ Cardiol 1990;66:1039-1044

22. Shaknovich A, Teirstein PS, Stratienko AA, Walker CM, Cleman MW, Schatz RA. Restenosis in single Palmaz-Schatz coronary stents: effects of prior PTCA and interval to prior PTCA (abstr). $J$ Am Coll Cardiol 1991;17:269A 23. Marco J, Fajadet J, Cassagneau B, Laurent JP, Robert G. Balloon expandable intracorondry stents: immediate and mean term results in a serie of 122 consecutive patients (abstr). Eur Heart $J$ 1990;11:2034A.

24. Holmes D, Schwartz R, Webster M. Coronary restenosis: what have we learned from angiography? $J$ Am Coll Cardiol 1991;17:14B-22B. 